Pacific Journal of Mathematic

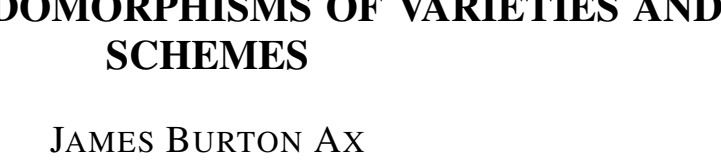




\title{
INJECTIVE ENDOMORPHISMS OF VARIETIES AND SCHEMES
}

\author{
JAMES AX
}

\begin{abstract}
It is shown that every injective endomorphism of a scheme $Y$ of finite type over a scheme $X$ is surjective. The proof is easily reduced to the case where $X$ is field which in turn follows from the analogous result for algebraic varieties. This result is proved using model theoretic methods to transfer the corresponding and trivially true fact about finite fields.
\end{abstract}

A finiteness property (Corollary 1 to the theorem) of algebraic varieties observed in $[1, \S 14]$ is that every injective endomorphism of a variety is surjective. Our purpose here is to establish a generalization of this result.

THEOREm. Let $Y$ be a scheme of finite type over a scheme $X$. Let $Y \stackrel{\varphi}{\longrightarrow} Y$ be an $X$-morphism. If $\varphi$ is injective then $\varphi$ is surjective.

COROLlary 1. Let $Y$ be an algebraic variety over an algebraically closed field $k$. Let $Y \stackrel{\varphi}{\longrightarrow} Y$ be a morphism. Assume that the induced mapping $\varphi(k)$ of the k-valued points $Y(k)$ of $Y$ to $Y(k)$ is injective. Then $\varphi(k)$ is surjective.

CoROLlary 2. Let $R \stackrel{\mu}{\longrightarrow} R$ be a homomorphism of a finitely generated algebra to itself. If for each prime ideal $p$ of $\mu(R)$ there is at most one prime $q$ of $R$ such that $q \cap \mu(R)=p$ then for each $p$ there is precisely one such $q$.

The proof of our main result goes through its Corollary 1 whose proof in [1] follows certain involved considerations about finite fields which, although they suggested the existence of such a result and motivated its proof, are completely unnecessary. We give afresh in $\S 1$ a brief proof of Corollary 1 . The main new point to be established is the special case of the theorem when $X=\operatorname{Spec}(k), k$ a field. This is accomplished by ascent to the algebraic closure.

Several possible extensions of the theorem suggest themselves. Of course there exist well-known examples of injective analytic endomorphisms which are not surjective, for example in [3, Chap. III, §1], an isomorphism of $\mathbf{C}^{2}$ onto an open nondense subset of $\mathbf{C}^{2}$ is defined. In $\S 4$ we exhibit a Dedekind domain not having the finiteness property. 
1. Injective endomorphisms of algebraic varieties. In this section, $k$ denotes an algebraically closed field. Let $Y$ be an algebraic variety and $Y \stackrel{\varphi}{\longrightarrow} Y$ a morphism, both defined over $k$. $\varphi$ defines a mapping (also called $\varphi$ ) from the underlying topological space of $Y$ (also called $Y$ ) to itself. $\phi$ also defines a map $\phi^{c}$ from the closed points $Y^{c}$ of $Y$ to $Y^{c}$. In Lemma 1 of $\S 2$ it is shown that $\varphi$ is injective (respectively: surjective) if and only if $\varphi^{c}$ is injective (respectively: surjective) . In the present situation $Y^{c}$ can be identified with the $k$-valued points $Y(k)$ of $Y$ over $k$.

Proof of Corollary 1. The complete case being trivial, the most interesting case is when $Y$ is affine; to simplify notation, we assume $Y$ affine (cf. the remark following the proof). Then there exist positive integers $n, t, d$ and polynomials $g_{1}, \cdots, g_{t}, f_{1}, \cdots, f_{n} \in k\left[X_{1}, \cdots, X_{n}\right]=$ $k[X]$ such that: $Y$ is (isomorphic to) $\operatorname{Spec}\left(k[X] /\left\langle g_{1}, \cdots, g_{t}\right\rangle\right) ; f_{1}, \cdots, f_{n}$ define a $k$-morphism $\operatorname{Spec}(k[X]) \rightarrow \operatorname{Spec}(k[X])$ inducing $Y \stackrel{\varphi}{\longrightarrow} Y$; $\operatorname{deg} g_{\text {г }}, \operatorname{deg} f_{\nu} \leqq d, \tau=1, \cdots, t, \nu=1, \cdots, n$.

Let $E=E_{n t d}$ be the following statement about an arbitrary field $K$ :

if $G_{1}, \cdots, G_{t}, F_{1}, \cdots, F_{n} \in K\left[X_{1}, \cdots, X_{n}\right]$ are such that

(a) $\operatorname{deg} G_{\mp}, \operatorname{deg} F_{\nu} \leqq d$ for $\tau=1, \cdots, t$ and $\nu=1, \cdots, n$; and

(b) if $x \in K^{n}$ is such that $G_{\tau}(x)=0$ for $\tau=1, \cdots, t$ then $G_{\tau}\left(F_{1}(x), \cdots, F_{n}(x)\right)=0$ for $\tau=1, \cdots, t$; and

(c) if $x, y \in K^{n}$ are such that $G_{\tau}(x)=G_{\tau}(y)=0$ for $\tau=1, \cdots, t$ and $F(x)=F_{\nu}(y)$ for $\nu=1, \cdots, n$ then $x=y$;

then for all $x \in K^{n}$ such that $G_{\tau}(x)=0$ for $\tau=1, \cdots, t$ there exists $w \in K^{n}$ such that $G_{\tau}(w)=0$ for $\tau=1, \cdots, t$ and such that $x_{\nu}=F_{\nu}(w)$, $\nu=1, \cdots, n$.

It suffices to prove that $E$ holds when $K$ is algebraically closed. Briefly, $E$ says that for all choices of $Y$ and of a polynomial mapping of $K^{n}$ to itself inducing $Y(K) \stackrel{\lambda}{\longrightarrow} Y(K)$ and for all $v \in Y(K)$ there exist $w, x, y \in Y(K)$ such that either $\lambda(w)=v$ or $\lambda(x)=\lambda(y)$. From this we see that $E$ is an elementary property, i.e., there exists an elementary statement $\mathscr{E}=\mathscr{E}_{n t d}$ such that $E$ holds for $K$ if and only if $\mathscr{E}$ is true in $K$. Moreover from our brief description of $E$ it is seen that $\mathscr{E}$ can be taken to be of universal-existential type, i.e., $\mathscr{P}$ is in the normal form $\forall X_{1} \cdots \forall X_{a} \exists X_{a+1} \cdots \exists X_{b} \mathscr{F}$ where $\mathscr{F}$ is quantifier free. This last fact means that if $E$ holds for each member of an ascending sequence of fields than $E$ holds for the union of the sequence. This is also easily verified directly.

$E$ is true when $K$ is finite since an injective mapping of a finite set to itself is surjective. Since the algebraic closure of a finite field is an ascending union of finite fields, $E$ is true when $K$ is the algebraic 
closure of a finite field. Now $E$ being an elementary statement is true in one algebraically closed field if and only if it is true every algebraically closed field of the same characteristic $[8, \S 5.8]$ or $[7$, $\S 9$, p. 109]. Thus $E$ is true in every algebraically closed field of prime characteristic. By a corollary $[7, \S 6, \mathrm{p} .111]$ to the compactness theorem [7, Proposition 2, p. 100], $E$ is true in every algebraically closed field, as desired. A more algebraic version of this proof would proceed by observing that $E$ is true for ultraproducts of fields for which it is true. Thus $E$ is true for every ultraproduct of the algebraic closures of finite fields. Since every algebraically closed field of cardinality the continuum can be so obtained $[7, \S 0, \mathrm{p} .67]$, the result follows again by the Lefshetz Principle.

REMARK. A detailed proof for $Y$ an abstract variety would be similar but with more complicated notation. It would necessitate considering a finite affine cover of $Y, Y=\bigcup_{i=1}^{a} Y_{i}$ with $Y_{i} \cap Y_{j}=Y_{i j}$ affine. Then we would need to consider affine imbeddings of the $Y_{i}$ and $Y_{i j}$ and polynomials defining the imbeddings as well as the maps including $\varphi$. Then there exists an elementary statement $E=E_{a d N}$ corresponding to the case where the above polynomials are all of degree at most $d$ in at most $N$ variables. The proof then continues as before.

2. Injective and surjective morphisms. Let $k$ be a field and let $V, W$ be schemes of finite type over $k$. Let $V^{c}$ denote the closed points of $V$. Any $\operatorname{Spec}(k)$-morphism $V \stackrel{\psi}{\longrightarrow} W$ induces a mapping $\psi^{c}=\left(\psi \mid V^{c}\right): V^{c} \rightarrow W^{c}$.

Lemma 1. $\psi$ is injective $\Leftrightarrow \psi^{c}$ is injective, $\psi$ is surjective $\Leftrightarrow \psi^{c}$ is surjective.

Proof. Let closures be denoted by bars. Let $v \in V, w \in Y$. Then $\psi(v)=w$ if and only if $\psi^{c}\left(\{\bar{v}\}^{c}\right)$ is dense in $\{\bar{w}\}^{c}$. The lemma is a straightforward consequence of this characterization of $\psi$ by $\psi^{c}$ and dimension theory.

We denote the function field of a variety $V$ by $K(V)$. If $Y$ is a scheme and $x$ is a point of $Y$ then $\kappa(x)$ is the residue class field of the local ring at $x$.

Lemma 2. Let $V \stackrel{\psi}{\longrightarrow} W$ be a Spec (k)-morphism of reduced and geometrically irreducible Spec(k)-schemes. Assume $\psi$ is dominating and injective. Then $\psi$ is purely inseparable.

Proof. The domination of $\psi$ allows us to regard $L=K(W)$ as a subfield of $N=K(V)$. The injectivity of $\psi$ implies that $N / L$ is a finite algebraic extension; we must show this extension is purely in- 
separable. By passage to affine opens we can assume $V$ and $W$ are affine: $V=\operatorname{Spec} B, W=\operatorname{Spec} A$. Let $M$ be the maximum separable extension of $L$ contained in $N$. There exists $\theta \in N$ such that $M=L(\theta)$. We can find an $a \in A-\{0\}$ with the following properties: $\theta \in B[1 / \alpha]$; $B[1 / a]$ is integral over $A[1 / a] ; A[1 / a]$ is normal; and $A[1 / a, \theta]$ is unramified over $A[1 / a]$. For all maximal ideals $p$ of $C=A[1 / a]$ we have

$$
[M: L]=\sum_{q}[\kappa(q): \kappa(p)]
$$

where the sum is over all maximal ideals $q$ of $D=C[\theta]$ for which $q \cap C=p$. Since $B[1 / a]$ contains $D$ and is integral over $C$, the injectivity of $\psi$ implies there is precisely one such maximal ideal $q_{p}$. To complete the proof it suffices to find $p$ such that $\left[\kappa\left(q_{p}\right): \kappa(p)\right]=1$. Let $f \in L[X]$ be the monic irreducible polynomial for $\theta$ over $L$. Since $C$ is normal, $f \in C[X]$.

We assert $f(C) \nsubseteq C^{*}$, i.e., that there exists $c \in C$ such that $f(c)$ is a nonunit of $C$, provided that $C \neq k$ (if $C=k$ then $L=M=k$ and there is nothing more to prove); i.e., provided $m=\operatorname{dim} W \geqq 1$. By Noether normalization, $C$ is a finite integral extension of $k[Y]=$ $k\left[Y_{1}, \cdots, Y_{m}\right]$. Let $\mathscr{N}$ denote the norm map $L \rightarrow k(Y)$. $\mathscr{N}$ defines multiplicative maps $C \rightarrow k[Y], C^{*} \rightarrow k[Y]^{*}=k^{*}$, and $C[X] \rightarrow k[Y][X]$. $g=\mathscr{N}(f)$ is monic polynomial in $X$ with coefficients in $k[Y]$. Hence there exists $c \in k[Y] \subseteq C$ such that $g(c) \in k[Y] \sim k^{*}$. Thus $\mathscr{N}(f(c))=$ $\mathscr{N}(f)(c)=g(c) \in k[Y] \sim k[Y]^{*}$. Thus $f(c) C \sim C^{*}$ which establishes our assertion.

Let $p$ be any maximal ideal containing $f(c)$. The $C$-homomorphism $C[X] \stackrel{\mu}{\longrightarrow} C$ defined by $\mu(X)=c$ composed with the natural surjection $C \stackrel{\nu}{\longrightarrow} \kappa(p)$ gives a $k$-homomorphism $C[X] \stackrel{\nu \circ \mu}{\longrightarrow} \kappa(p)$ with kernel $W$ generated by $X-c$ and $p$. Since $f(c) \in p, f(X) \in W$ and so $\nu \circ \mu$ defines a $k$-homomorphism $D=C[X] /(f) \stackrel{\rho}{\longrightarrow} \kappa(p)$. The kernel of $\rho$ is a maximal ideal of $D$ above $p$, i.e., $q_{p}$ and $\rho$ induces an inverse of the natural inclusion $\kappa(p) \subset \kappa\left(q_{p}\right)$. This completes the proof.

REMARK. The case of Lemma 2 corresponding to $k$ algebraically closed is well-known [5, Th. 3, p. 115]. In the general case the crucial point is to establish the existence of a closed point of degree 1 . The existence of a closed point of degree 1 can actually be established in greater generality. For example, if $V \stackrel{\psi}{\longrightarrow} W$ is any nonconstant $k$ morphism of algebraic varieties (defined over any field $k$ ), then there exists closed points $q$ of $V$ and $p$ of $W$ such that $\psi(q)=p$ and $\kappa(q) \approx_{k} \kappa(p)$.

3. Reduction to varieties. In this section we show how the 
theorem follows from the special case where $X=\operatorname{Spec}(k), k$ a perfect field and where $Y$ is variety defined over $k$, i.e., $Y$ is a reduced and geometrically irreducible scheme of finite type over $k$.

Let $x$ be a point of $X, O_{x}$ its local ring and $\kappa(x)$ the residue class field of $O_{x}$. Let $F_{x}$ be the fibre of $Y \stackrel{\pi}{\longrightarrow} X$ above $x ; F_{x}$ can be defined as the fibre product of $\pi$ and the canonical map Spec $(\kappa(x)) \rightarrow X$. Thus we get a universal commutative diagram

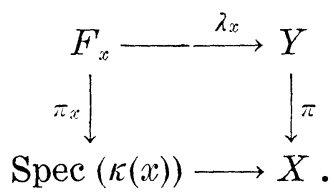

The map $\lambda_{x}$ is a bijection of the points of $F_{x}$ onto the points $y \in Y$ for which $\pi(y)=x$. The commutative diagram
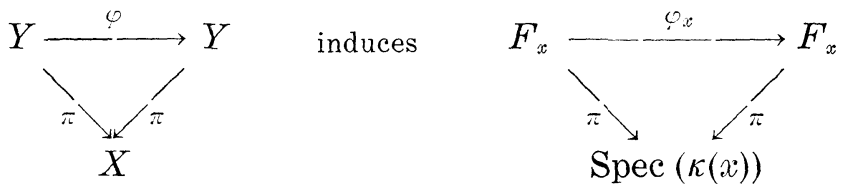

and since $\varphi$ is injective so is $\varphi_{x}$. Using that $Y \stackrel{\pi}{\longrightarrow} X$ is of finite type we deduce that $\pi_{x}$ is of finite type; indeed the only finiteness condition we need about $\pi$ is that $\pi_{x}$ is of finite type for all $x \in X$.

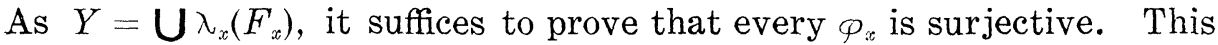
shows that it suffices to prove the main theorem in the special case where $X=\operatorname{Spec}(k), k$ a field.

Let $\sqrt{k}$ denote the perfect closure of $k$. Then

$$
X x_{\text {Spec }(k)} \operatorname{Spec}(\sqrt{k}) \longrightarrow X
$$

is bijective so that we can assume $k$ perfect. If $Y_{\text {red }} \stackrel{j}{\longrightarrow} Y$ is the canonical map of the (maximal) closed reduced subscheme of $Y$ to $Y$ then $j$ is a bijection so that we can assume $Y$ is reduced.

We now show that we can assume that $Y$ is irreducible over $k$, and hence a $k$-variety. Let $Y=\mathrm{U}_{\tau=1}^{t} Y_{\tau}$ where the $Y_{\text {: }}$ are $k$-varieties. We proceed by induction on $d=\operatorname{dim} Y=\max _{-} \operatorname{dim} Y_{-}$. We arrange notation so that $Y_{1}, \cdots, Y_{s}$ are all the components of dimension $d$. Since $\varphi$ is injective, $\varphi\left(\bigcup_{o=1}^{s} Y_{\sigma}\right) \subseteq \bigcup_{o=1}^{s} Y_{\sigma}$. For all $\sigma \in[1, s]$ as $Y_{\sigma}$ is irreducible there exists $p(\sigma) \in[1, s]$ such that $\phi\left(Y_{\sigma}\right) \subseteq Y_{p(\sigma)}$. The mapping $[1, s] \stackrel{p}{\longrightarrow}[1, s]$ is, by dimension theory (cf. $\S 2$ ) and the injectivity of $\varphi$, itself an injection, i.e., a permutation of $[1, s]$. Thus there exists a positive integer $e$ such that $p^{(e)}$ is the trivial permutation of $[1, s]$. Replacing $\rho$ by $\varphi^{(e)}$ we have that $p$ is already trivial. Thus $\left(\varphi \mid Y_{\sigma}\right): Y_{\sigma} \rightarrow Y_{\sigma}$ is injectve. Hence assuming the theorem established 
for $k$-varieties we have that each $\left(\varphi \mid Y_{\sigma}\right)$ is surjective for $\sigma \in[1, s]$. This implies that $\phi\left(\mathrm{U}_{\tau=s+1}^{t} Y_{\tau}\right) \subseteq \bigcup_{\tau=s+1}^{t} Y_{\tau}$ and so by inductive hypothesis, $\varphi\left(\bigcup_{\tau=s+1}^{t} Y_{\tau}\right)=\bigcup_{\tau=s+1}^{t} Y_{\tau}$. Therefore $\varphi$ is surjective.

Still assuming $k$ is perfect let $Y \stackrel{\pi}{\longrightarrow}$ Spec $(k)$ with $Y$ a $k$-variety. Let $k^{\prime}$ denote the relative algebraic closure of $k$ in $K(Y)$, the function field of $Y$. Then we have the factorization $[Y \rightarrow \operatorname{Spec}(k)]=$ $\left[Y \stackrel{\pi^{\prime}}{\longrightarrow} \operatorname{Spec}\left(k^{\prime}\right) \rightarrow \operatorname{Spec}(k)\right]$. Here $Y \stackrel{\pi^{\prime}}{\longrightarrow}$ Spec $\left(k^{\prime}\right)$ gives $Y$ the structure of a variety defined over $k^{\prime}$ but $Y \stackrel{\varphi}{\longrightarrow} Y$ need not be a Spec $\left(k^{\prime}\right)$ morphism. Nevertheless $\phi$ induces a $k$-automorphism $\alpha$ of $k^{\prime}$. Since $\left[k^{\prime}: k\right]<\infty$ there exists a positive integer $f$ such that $\alpha^{f}$ is trivial on $k^{\prime}$. Then $\varphi^{f}$ is a Spec $\left(k^{\prime}\right)$-morphism and our reduction is complete.

4. Completion of the proof. We now complete the proof of the main theorem by proving it when $Y$ is an algebraic variety defined over a field $k$ and $\psi$ is a $k$-morphism. Let $Y^{\prime}$ denote the normalization of $Y x_{\text {spec }(k)} \operatorname{Spec}(\tilde{k})$ where $\widetilde{k}$ is an algebraic closure of $k$. We have a commutative diagram:

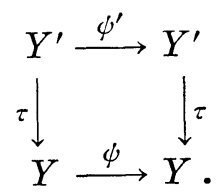

Since $\tau$ is surjective with finite fibres and $\psi$ is injective, $\psi^{\prime}$ has finite fibres. By Zariski's Main Theorem [6, p. 414], there exists a factorization $Y^{\prime} \subset Z \stackrel{\sigma}{\longrightarrow} Y^{\prime}$ of $\psi$ where $\sigma$ is finite and $Y^{\prime} \subset Z$ is an isomorphism of $Y^{\prime}$ onto a (dense) open of $Z$. By Lemma $2, \psi$ is purely inseparable. Hence so are $\psi^{\prime}$ and $\sigma$. As $Y^{\prime}$ is normal, $\sigma$ is a bijection. Thus $\psi^{\prime}$ is injective. By the corollary to main theorem which we have already established in $\S 1, \psi^{\prime}$ is surjective. $\tau$ is also surjective and hence so is $\psi$. This completes the proof.

An Example. The implication "one-one implies onto" seems to be a persistent property of self-mappings of objects with "some finiteness" conditions. Indeed this property is for abstract sets the definition of finite. The "first" set not satisfying this is the positive integers $\mathbf{P}$ with the mapping $p \rightarrow p+1$. This can be made into an example of a nice Noetherian ring $R$ and a homomorphism $R \stackrel{\varphi}{\longrightarrow} R$ which gives a nonsurjective injection $\operatorname{Spec}(R) \rightarrow \operatorname{Spec}(R)$. Indeed, let $R=$ $\mathbf{C}\left[t,(t-c)^{-1}: c \in \mathbf{C} \sim \mathbf{P}\right]$ and let $\varphi$ be defined by $t \rightarrow t+1$. Then as point sets, $\mathbf{P}=\operatorname{Spec}(R)$ and the mapping is as above. Since $R$ is the intersection of the discrete valuation rings $\mathrm{C}[t]_{q}$ where $q$ runs through the prime ideals $(t-c) \mathbf{C}[t]$ for $c \in \mathbf{P}, R$ is a Dedekind domain. 
We would like to mention some recent proofs of these results. In conversation, G. Shimura showed how to obtain Corollary 1 by using reduction modulo $p$ to again reduce the result to the case where $k$ is the algebraic closure of a finite field. It seems that this technique would also be able to directly establish the case of the main result where $X=\operatorname{Spec}(k), k$ a field (from which the theorem is easily deduced). A third proof was given by A. Borel [4]. His proof is cohomological and proves Corollary 1 at least in characteristic zero. While the prime characteristic case would be difficult to establish by this technique in complete generality, Borel was able to extend his method to prove a real analogue of Corollary 1. More recently S. Lichtenbaum gave a direct proof of the theorem when $X=\operatorname{Spec}(k), k$ a field and $Y$ is affine using the Mordell-Weil Theorem. Finally we should mention that the first and only previous result of this kind was obtained by A. BialynickiBirula and M. Rosenlicht who gave a simple proof in [2] of the special case of Corollary 1 when $Y$ is affine space $\mathbf{A}^{n}$.

We would like to thank S. Lichtenbaum for his helpful discussion of several points.

\section{REFERENCES}

1. J. Ax, The elementary theory of finite fields, Ann. of Math. 88 (1968), 239-271.

2. A. Bialynicki-Birula and M. Rosenlicht, Injective morphisms of algebraic varieties, Proc. Amer. Math. Soc. 13 (1962), 200-203.

3. S. Bochner and W. T. Martin, Several complex variables, Princeton University Press, 1948.

4. A. Borel, Injective endomorphisms of algebraic varieties (to appear).

5. C. Chevalley, Fondements de la geometrie algebrique, Fac. des Sciences de Paris, 1958.

6. D. Mumford, Introduction to algebraic geometry, Lecture Notes, Harvard University, 1966.

7. P. Ribenboim, La conjecture d'Artin sur les equations diophantiennes, Queen's Papers on Pure and Applied Mathematics, No. 14, Queen's Univ., Kingston, Ontario, 1968.

8. A. Robinson, On the metamathematics of algebra, North Holland, Amsterdam, 1951.

Received December 6, 1968. During work on this paper the author was a Sloan Fellow and was partially supported by NSF Grant GP-7945.

CORNELL UNIVERSITY 



\section{PACIFIC JOURNAL OF MATHEMATICS}

\section{EDITORS}

H. ROYDEN

Stanford University

Stanford, California

Richard Pierce

University of Washington

Seattle, Washington 98105
J. DUGUNDJI

Department of Mathematics

University of Southern California

Los Angeles, California 90007

BASIL GORDON

University of California

Los Angeles, California 90024

\section{ASSOCIATE EDITORS}
E. F. BECKENBACH
B. H. NEUMANN
F. WOLF
K. YosHIDA

\section{SUPPORTING INSTITUTIONS}

UNIVERSITY OF BRITISH COLUMBIA

CALIFORNIA INSTITUTE OF TECHNOLOGY

UNIVERSITY OF CALIFORNIA

MONTANA STATE UNIVERSITY

UNIVERSITY OF NEVADA

NEW MEXICO STATE UNIVERSITY

OREGON STATE UNIVERSITY

UNIVERSITY OF OREGON

OSAKA UNIVERSITY

UNIVERSITY OF SOUTHERN CALIFORNIA
STANFORD UNIVERSITY

UNIVERSITY OF TOKYO

UNIVERSITY OF UTAH

WASHINGTON STATE UNIVERSITY

UNIVERSITY OF WASHINGTON

*

AMERICAN MATHEMATICAL SOCIETY CHEVRON RESEARCH CORPORATION TRW SYSTEMS

NAVAL WEAPONS CENTER 


\section{Pacific Journal of Mathematics}

\section{Vol. 31, No. $1 \quad$ November, 1969}

James Burton Ax, Injective endomorphisms of varieties and schemes........

Richard Hindman Bouldin, A generalization of the Weinstein-Aronszajn

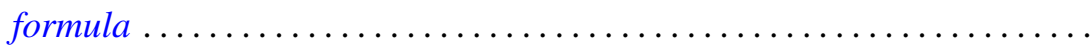

John Martin Chadam, The asymptotic behavior of the Klein-Gordon equation with external potential. II ...............................

Rina Hadass, On the zeros of the solutions of the differential equation

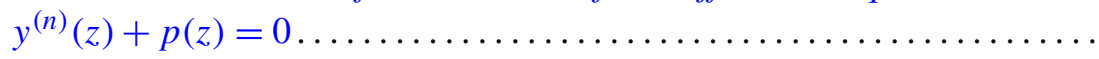

John Sollion Hsia, Integral equivalence of vectors over local modular lattices. II .............................................

Robert Hughes, Boundary behavior of random valued heat polynomial

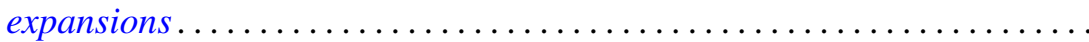

Surender Kumar Jain, Saad H. Mohamed and Surjeet Singh, Rings in which every right ideal is quasi-injective .........................

T. Kawata, On the inversion formula for the characteristic function .........

Erwin Kleinfeld, On right alternative rings without proper right ideals......

Robert Leroy Kruse and David Thomas Price, On the subring structure of

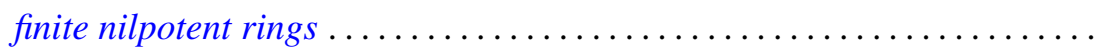

Marvin David Marcus and Stephen J. Pierce, Symmetric positive definite multilinear functionals with a given automorphism. .

William Schumacher Massey, Pontryagin squares in the Thom space of a bundle...

William Schumacher Massey, Proof of a conjecture of Whitney ...

John William Neuberger, Existence of a spectrum for nonlinear transformations

Stephen E. Newman, Measure algebras on idempotent semigroups ...

$\mathrm{K}$. Chandrasekhara Rao, Matrix transformations of some sequence spaces

Robert Bruce Schneider, Some theorems in Fourier analysis on symmetric

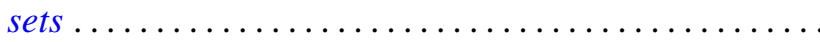

Ulrich F. K. Schoenwaelder, Centralizers of abelian, normal subgroups of hypercyclic groups...

Jerrold Norman Siegel, $G$-spaces, $H$-spaces and $W$-spaces

Robert Irving Soare, Cohesive sets and recursively enumerable Dedekind cuts...

Kwok-Wai Tam, Isometries of certain function spaces .... . .

Awadhesh Kumar Tiwary, Injective hulls of semi-simple modules over regular rings ....

Eldon Jon Vought, Concerning continua not separated by any nonaposyndetic subcontinuum .................... 